\title{
Perception of Radiation Risk as a Predictor of Mid-Term Mental Health after a Nuclear Disaster: The Fukushima Health Management Survey
}

\author{
Itaru Miura ${ }^{1, *}$, Masato Nagai ${ }^{2,3}$, Masaharu Maeda ${ }^{2,4}$, Mayumi Harigane ${ }^{2}$, Senta Fujii ${ }^{2,4}$, \\ Misari Oe ${ }^{5}$ (D), Hirooki Yabe ${ }^{1}$ (D), Yuriko Suzuki ${ }^{6}$ (D), Hideto Takahashi ${ }^{2}$, Tetsuya Ohira ${ }^{2,3}$, \\ Seiji Yasumura ${ }^{2,7}$ and Masafumi Abe ${ }^{2}$ \\ 1 Department of Neuropsychiatry, School of Medicine, Fukushima Medical University, Fukushima 960-1295, \\ Japan; hyabe@fmu.ac.jp \\ 2 Radiation Medical Science Center for the Fukushima Health Management Survey, Fukushima 960-1295, \\ Japan; mnagai@fmu.ac.jp (M.N.); masagen@fmu.ac.jp (M.M.); harigane@fmu.ac.jp (M.H.); \\ fuji-sen@fmu.ac.jp (S.F.); htaka@fmu.ac.jp (H.T.); fwge1119@mb.infoweb.ne.jp (T.O.); \\ yasumura@fmu.ac.jp (S.Y.); masafumi@fmu.ac.jp (M.A.) \\ 3 Department of Epidemiology, School of Medicine, Fukushima Medical University, \\ Fukushima 960-1295, Japan \\ 4 Department of Disaster Psychiatry, School of Medicine, Fukushima Medical University, \\ Fukushima 960-1295, Japan \\ 5 Department of Neuropsychiatry, School of Medicine, Kurume University, Fukuoka 830-0011, Japan; \\ oe_misari@kurume-u.ac.jp \\ 6 Department of Adult Mental Health, National Institute of Mental Health, National Center of Neurology and \\ Psychiatry, Tokyo 187-8553, Japan; yrsuzuki@ncnp.go.jp \\ 7 Department of Public Health, School of Medicine, Fukushima Medical University, \\ Fukushima 960-1295, Japan \\ * Correspondence: itaru@fmu.ac.jp; Tel.: +81-24-547-1331, Fax: +81-24-548-6735
}

Received: 2 August 2017; Accepted: 12 September 2017; Published: 15 September 2017

\begin{abstract}
Predictive factors including risk perception for mid-term mental health after a nuclear disaster remain unknown. The purpose of this study was to examine the association between perceived radiation risk and other factors at baseline and mid-term mental health after the Fukushima Daiichi nuclear disaster of 2011 in Japan. A mail-based questionnaire survey was conducted in January 2012 and January 2013. Mental health status was assessed using the K6 scale. Psychological distress over the 2-year period was categorized into the following four groups: chronic, recovered, resistant, or worsened. Most participants (80.3\%) were resistant to the disaster. A positive association was found between the radiation risk perception regarding immediate effects and the worsened group in women. Baseline post-traumatic stress disorder (PTSD) or a history of psychiatric disease predicted being in the chronic or worsened group in mid-term course. These results suggest that evacuees who believed that their health was substantially affected by the nuclear disaster were at an increased risk of having poor mid-term mental health in women. Careful assessment of risk perception after a nuclear disaster, including the presence of PTSD or a history of psychiatric disease, is needed for appropriate interventions.
\end{abstract}

Keywords: risk perception; nuclear disaster; mid-term mental health; evacuation; predictive factor

\section{Introduction}

Although post-traumatic stress disorder (PTSD) is the most common psychiatric reaction to disaster, depression, which often coexists with PTSD [1], is also important because it may lead to 
suicidal ideation. Because the prevalence of psychological morbidity including depression and anxiety typically decrease after a disaster [2-4], being able to predict the worsening of mental health problems among sufferers is important in order to provide appropriate care and support from the early phase.

Many studies have reported risk factors for mental health after disasters, suggesting that disaster exposure [5-7], general neurotic personality [5], aging, female sex, job stress [8], and baseline PTSD [9] predicted poor mid-term mental health. A recent systematic review [10] showed that various factors were associated with mental health status; i.e.; (1) factors related to the disaster (e.g., higher level of exposure; those who were evacuated; those who suffered financial losses; loss of employment), (2) coping factors (e.g., positive and proactive behaviors), (3) health-related factors (e.g., poor mental or physical health status before disaster), (4) personal factors (e.g., female or male gender; older age; lower the socioeconomic status). Moreover, a recent meta-analysis [11] suggested that predictors for depression after disasters included both pre-existing personal factors (female gender, not married; holding religious beliefs; poor education; prior trauma) and disaster-related factors (disaster experience, loss of employment or property, suffering house damage).

After the Chernobyl nuclear power plant accident in 1986, exposure to a nuclear disaster [12], female sex [13], close proximity to the disaster site [14,15], perception of radiation risk [15], and being an evacuee $[16,17]$ were all associated with poor long-term mental health. However, no well-designed longitudinal studies were conducted in the first three years after the accident. The Fukushima Daiichi nuclear disaster resulting from the Great East Japan Earthquake on 11 March 2011, forced many people in Fukushima Prefecture to evacuate from their hometowns. A recent cross-sectional study [18] reported that the perception of radiation risk was associated with psychological distress after the disaster. Risk perception is important as a cognitive factor, and a cognitive model of PTSD [19] suggested that PTSD becomes persistent when individuals process the trauma in a way that leads to a sense of serious, current threat. Previous studies showed that perception of risk was associated with mental health problems such as PTSD, acute stress disorder, and depression after nuclear [15,20-22] or non-nuclear [23,24] disaster. Moreover, incorrect understanding of effects of radiation on health was associated with bad mental health status in Nagasaki, Japan [25]. Although risk perception is influenced by disaster experiences [26], it has been suggested that perception of risk may have considerable effects on mental health status, in particular after nuclear accident. Because there are no well-designed longitudinal studies regarding associations between perceived radiation risk and other predictive factors at baseline and mid-term mental health problems after a nuclear disaster, we examined the association between the perception of radiation risk and other predictive factors at baseline and mid-term mental health after the Fukushima Daiichi nuclear disaster using longitudinal data from the large-scale Fukushima Health Management Survey [27].

\section{Materials and Methods}

\subsection{Participants}

The Fukushima Health Management Survey is a mail-based questionnaire survey focusing on lifestyle and mental health among adult residents (aged $\geq 15$ years on 11 March 2011) of the evacuation zone designated by the Japanese government (i.e., Hirono town, Naraha town, Tomioka town, Kawauchi village, Okuma town, Futaba town, Namie town, Katsurao village, Minamisoma city, Tamura city, Kawamata town, Iitate village, and part of Date city in Fukushima Prefecture) $[27,28]$ that was first carried out in fiscal year (FY) 2011 (January 2012, 10 months after the disaster). A follow-up survey was conducted in FY2012 (January 2013). There were 180,604 targeted residents of the evacuation zone in FY2011 and 184,507 in FY2012. Figure S1 shows a flowchart of the study selection and inclusion procedures. No responses were received from 107,171 and 32,220 residents in FY2011 and FY2012, respectively. In addition, 5157 respondents were excluded from analysis because of missing data. Finally, 36,056 participants were included in the analysis. 


\subsection{Measures}

The outcome of this study was scores on the K6 scale [29], which is composed of six questions (score range: 0-24) that assess non-specific psychological distress during the previous 30 days. The Japanese version of the K6 scale has been validated [30]. In this study, we adopted the cut-off score of $\geq 13$, which is classified as indicating probable severe mental illness [31]. The PTSD Checklist Stressor-Specific Version (PCL-S) [32] was used to assess post-traumatic symptoms. The PCL-S is a 17-item self-report measure, and the Japanese version has been shown to have sufficient validity and reliability [33]. The cut-off was set as a PCL-S score of $\geq 44$ [32]. Data was collected on the participants' sociodemographic characteristics, including age, sex, education, and living status, as well as subjective health status, perceived radiation risk, and disaster experiences and history. With regard to perceived radiation risk, we asked participants about their beliefs on the potential risks of radiation exposure [34] using the following questions: (i) "What is the likelihood of suffering immediate health damage (e.g., dying within 1 month) as a result of your current level of radiation exposure?"; (ii) What is the likelihood of damage to your health (e.g., cancer onset) in later life as a result of your current level of radiation exposure?"; and (iii) What is the likelihood that the health of your future (i.e., as of yet unborn) children and grandchildren will be affected as a result of your current level of radiation exposure?" Participants were asked to respond to each question using a 4-point Likert scale (1, very unlikely; 2, unlikely; 3, likely; 4, very likely). These items were translated into Japanese, then back to English, and modified after discussion with the authors of the questionnaire. We sent reminders to non-responders to the survey, but there were no incentives for the participants. This study was approved by the ethics committee of Fukushima Medical University (No. 1316). We obtained written informed consent from guardians on behalf of the children enrolled in the study to conduct an epidemiologic study based on the guidelines of the Council for International Organizations of Medical Science [35]. The study was conducted in accordance with the approved guidelines.

\subsection{Statistical Analysis}

Only the participants who responded to the K6 scale in both FY2011 and FY2012 were included in the analysis. Based on K6 scores from both years, we divided mental health status over the 2-year period into the following four groups: chronic group (K6 score $\geq 13$ in both FY2011 and FY2012); recovered group (K6 score $\geq 13$ in FY2011 and $<13$ in FY2012); resistant group (K6 score $<13$ in both FY2011 and FY2012); and worsened group (K6 score < 13 in FY2011 and $\geq 13$ in FY2012). To investigate predictive factors for mid-term mental health status, we focused the changes in psychological distress (K6) from baseline. We used logistic regression analysis to investigate the predictive factors at baseline associated with: (1) worsened group (compared with resistant group; K6 score < 13 at baseline in both groups) and (2) recovered group (compared with chronic group; $\mathrm{K} 6$ score $\geq 13$ at baseline in both groups). Multivariate logistic regression analysis adjusting all predictors (age, subjective health status, past history, disaster experiences, living place and arrangement in 2011, posttraumatic stress symptoms in 2011, and radiation risk perception in 2011 (immediate, delayed, and genetic effects)) were used. We analyzed males and females separately because gender may have effects on mental health status after disaster [11,36,37]. All statistical analyses were conducted using SAS (version 9.3; SAS Institute Inc., Cary, NC, USA).

\section{Results}

\subsection{Participant Characteristics}

The baseline characteristics of the four groups are shown in Table 1. For the total population, the mean scores ( \pm standard deviation) on the K6 scale were $6.2 \pm 5.7$ in FY2011 and $5.7 \pm 5.5$ in FY2012. 
Table 1. Baseline characteristics of each group in 2011.

\begin{tabular}{|c|c|c|c|c|}
\hline & Chronic & Recovered & Resistant & Worsened \\
\hline & $\begin{array}{c}(\mathrm{K} 6 \geq 13 \Rightarrow \\
\mathrm{K} 6 \geq 13)\end{array}$ & $\begin{array}{c}(\mathrm{K} 6 \geq 13 \Rightarrow \\
\mathrm{K} 6<13)\end{array}$ & $\begin{array}{c}(\mathrm{K} 6<13 \Rightarrow \\
\mathrm{K} 6<13)\end{array}$ & $\begin{array}{c}(K 6<13 \Rightarrow \\
K 6 \geq 13)\end{array}$ \\
\hline \multicolumn{5}{|l|}{ Men } \\
\hline No. of participants & 790 & 995 & 12,911 & 757 \\
\hline Mean age (years) & $57.7 \pm 16.4$ & $58.0 \pm 16.4$ & $57.9 \pm 16.9$ & $57.9 \pm 17.6$ \\
\hline $15-39$ years $(\%)$ & 17.0 & 17.4 & 17.6 & 19.7 \\
\hline $40-64$ years $(\%)$ & 45.4 & 44.0 & 43.8 & 39.0 \\
\hline$\geq 65$ years $(\%)$ & 37.6 & 38.6 & 38.6 & 41.4 \\
\hline $\begin{array}{l}\text { Subjective health status } \\
\text { bad or very bad (\%) }\end{array}$ & 60.7 & 44.9 & 12.4 & 32.4 \\
\hline Disaster experiences & & & & \\
\hline Tsunami (\%) & 31.4 & 31.5 & 23.6 & 28.1 \\
\hline $\begin{array}{l}\text { Heard the sound of nuclear plant } \\
\text { explosion }(\%)\end{array}$ & 70.6 & 68.7 & 55.7 & 66.6 \\
\hline \multicolumn{5}{|l|}{ Bereavement } \\
\hline Yes $(\%)$ & 30.3 & 28.5 & 18.4 & 23.5 \\
\hline \multicolumn{5}{|l|}{ House damage } \\
\hline No damage (\%) & 16.2 & 17.6 & 26.9 & 21.3 \\
\hline Partial collapse $(\%)$ & 66.6 & 69.5 & 65.3 & 68.6 \\
\hline Half collapse and worse (\%) & 17.3 & 12.9 & 7.9 & 10.1 \\
\hline \multicolumn{5}{|l|}{ Past history } \\
\hline $\begin{array}{l}\text { Cardiovascular disease (heart } \\
\text { disease and stroke) }(\%)\end{array}$ & 23.0 & 22.4 & 14.5 & 18.0 \\
\hline Psychiatric disease $(\%)$ & 20.8 & 12.9 & 2.8 & 8.3 \\
\hline \multicolumn{5}{|l|}{ Living place } \\
\hline Out of Fukushima prefecture (\%) & 25.8 & 20.9 & 16.7 & 22.6 \\
\hline Living arrangement & & & & \\
\hline Other than own home $(\%)$ & 80.1 & 76.9 & 64.1 & 77.0 \\
\hline \multicolumn{5}{|l|}{ Posttraumatic stress symptoms } \\
\hline PCL-S score $\geq 44(\%)$ & 81.0 & 68.8 & 8.9 & 36.2 \\
\hline \multicolumn{5}{|l|}{ Radiation Risk Perception } \\
\hline \multicolumn{5}{|l|}{ Immediate effect } \\
\hline Very unlikely (\%) & 43.4 & 48.0 & 71.9 & 58.0 \\
\hline Unlikely (\%) & 23.7 & 22.5 & 17.9 & 21.0 \\
\hline Likely (\%) & 14.3 & 14.2 & 5.7 & 10.8 \\
\hline Very likely (\%) & 18.6 & 15.3 & 4.5 & 10.2 \\
\hline \multicolumn{5}{|l|}{ Delayed effect } \\
\hline Very unlikely (\%) & 11.0 & 13.8 & 29.7 & 17.7 \\
\hline Unlikely (\%) & 18.7 & 20.3 & 32.2 & 25.9 \\
\hline Likely (\%) & 24.2 & 24.4 & 19.7 & 23.9 \\
\hline Very likely (\%) & 46.1 & 41.5 & 18.5 & 32.5 \\
\hline \multicolumn{5}{|l|}{ Genetic effect } \\
\hline Very unlikely (\%) & 7.1 & 9.5 & 20.8 & 12.1 \\
\hline Unlikely (\%) & 12.2 & 14.1 & 27.8 & 20.0 \\
\hline Likely (\%) & 20.2 & 23.0 & 24.0 & 23.8 \\
\hline Very likely (\%) & 60.5 & 53.4 & 27.3 & 44.2 \\
\hline \multicolumn{5}{|l|}{ Women } \\
\hline No. of participants & 1464 & 1748 & 15,183 & 1091 \\
\hline Mean age (years) & $58.6 \pm 17.6$ & $57.1 \pm 17.4$ & $54.5 \pm 17.9$ & $57.4 \pm 18.5$ \\
\hline $15-39$ years $(\%)$ & 18.0 & 19.8 & 25.2 & 21.5 \\
\hline $40-64$ years $(\%)$ & 42.7 & 44.2 & 43.5 & 40.2 \\
\hline$\geq 65$ years $(\%)$ & 39.3 & 36.0 & 31.3 & 38.4 \\
\hline
\end{tabular}


Table 1. Cont.

\begin{tabular}{|c|c|c|c|c|}
\hline & Chronic & Recovered & Resistant & Worsened \\
\hline & $\begin{array}{c}(\mathrm{K} 6 \geq 13 \Rightarrow \\
\mathrm{K} 6 \geq 13)\end{array}$ & $\begin{array}{c}(\mathrm{K} 6 \geq 13 \Rightarrow \\
\mathrm{K} 6<13)\end{array}$ & $\begin{array}{c}(\mathrm{K} 6<13 \Rightarrow \\
\mathrm{K} 6<13)\end{array}$ & $\begin{array}{c}(\mathrm{K} 6<13 \Rightarrow \\
\mathrm{K} 6 \geq 13)\end{array}$ \\
\hline $\begin{array}{l}\text { Subjective health status } \\
\text { bad or very bad (\%) }\end{array}$ & 61.7 & 43.3 & 12.1 & 33.8 \\
\hline $\begin{array}{c}\text { Disaster experiences } \\
\text { Tsunami }(\%) \\
\text { Heard the sound of nuclear plant } \\
\text { explosion }(\%)\end{array}$ & $\begin{array}{l}22.5 \\
69.3\end{array}$ & $\begin{array}{l}24.7 \\
66.5\end{array}$ & $\begin{array}{l}16.4 \\
48.8\end{array}$ & $\begin{array}{l}21.6 \\
63.0\end{array}$ \\
\hline $\begin{array}{l}\text { Bereavement } \\
\text { Yes }(\%)\end{array}$ & 33.3 & 30.3 & 18.6 & 24.3 \\
\hline $\begin{array}{c}\text { House damage } \\
\text { No damage }(\%) \\
\text { Partial collapse }(\%) \\
\text { Half collapse and worse }(\%)\end{array}$ & $\begin{array}{l}19.6 \\
66.7 \\
13.8\end{array}$ & $\begin{array}{l}20.0 \\
68.7 \\
11.3\end{array}$ & $\begin{array}{c}30.4 \\
61.6 \\
8.0\end{array}$ & $\begin{array}{l}20.6 \\
66.4 \\
13.0\end{array}$ \\
\hline $\begin{array}{c}\text { Past history } \\
\text { Cardiovascular disease (heart } \\
\text { disease and stroke) }(\%)\end{array}$ & 18.3 & 14.1 & 8.1 & 15.8 \\
\hline Psychiatric disease (\%) & 24.8 & 12.7 & 3.3 & 11.4 \\
\hline \multicolumn{4}{|l|}{ Living place } & 23.0 \\
\hline $\begin{array}{l}\text { Living arrangement } \\
\text { Other than own home (\%) }\end{array}$ & 81.8 & 76.3 & 66.7 & 76.3 \\
\hline \multicolumn{5}{|l|}{ Posttraumatic stress symptoms } \\
\hline \multicolumn{5}{|l|}{ Radiation Risk Perception } \\
\hline \multicolumn{5}{|l|}{ Immediate effect } \\
\hline Very unlikely (\%) & 46.6 & 50.7 & 70.6 & 55.7 \\
\hline Unlikely (\%) & 22.9 & 24.4 & 19.0 & 23.7 \\
\hline Likely (\%) & 15.4 & 12.7 & 6.6 & 11.4 \\
\hline Very likely (\%) & 15.1 & 12.2 & 3.9 & 9.2 \\
\hline \multicolumn{5}{|l|}{ Delayed effect } \\
\hline Very unlikely (\%) & 9.5 & 10.4 & 22.5 & 14.9 \\
\hline Unnlikely (\%) & 18.5 & 22.2 & 33.0 & 24.6 \\
\hline Likely $(\%)$ & 26.2 & 26.6 & 24.2 & 29.3 \\
\hline Very likely (\%) & 45.8 & 40.9 & 20.4 & 31.2 \\
\hline \multicolumn{5}{|l|}{ Genetic effect } \\
\hline Very unlikely (\%) & 5.2 & 5.6 & 15.0 & 10.0 \\
\hline Unlikely (\%) & 11.5 & 13.7 & 28.0 & 16.4 \\
\hline Likely (\%) & 21.7 & 26.3 & 28.0 & 28.3 \\
\hline Very likely (\%) & 61.6 & 54.4 & 29.1 & 45.3 \\
\hline
\end{tabular}

PCL-S, Post-Traumatic Checklist Stressor-Specific Version.

The prevalence of being at high risk for psychological distress (K6 score $\geq 13$ ) was $14.3 \%$ in FY2011 and 11.7\% in FY2012, which are relatively high compared with the data of non-disaster settings in Japan (4.7\%, among 15-64 years old) [38]. In those who responded in only FY2011 (and did not respond in FY2012), the prevalence of K6 score $\geq 13$ was 15.2\% in FY2011, and was slightly higher than those who respond in both FY2011 and FY 2012. The whole sample was categorized according to the definition stated above as follows: chronic group (6.5\%); recovered group (7.8\%); resistant group $(80.3 \%)$; and worsened group (5.3\%).

\subsection{Predictors for The Worsened Group Compared with the Resistant Group}

Table 2 shows the results of univariate and multivariate analyses of potential factors in the worsened group compared with the resistant group (the results in total subjects are shown in Table S1). In univariate analysis, the perception that immediate (odds ratio [OR]: $2.82,95 \%$ confidence interval [CI]: 2.18-3.64 in men; OR: 2.97, 95\% CI: 2.36-3.73 in women), delayed (OR: 2.96, 95\% CI: 2.38-3.67 
in men; OR: $2.31,95 \%$ CI: 1.90-2.80 in women), or genetic (OR: 2.78, 95\% CI: 2.19-3.52 in men; OR: $2.33,95 \%$ CI: $1.88-2.89$ in women) radiation effects were very likely was positively associated with the worsened group (Table 2). Similarly, the perception that immediate (OR: 2.33, 95\% CI: 1.82-2.99 in men; OR: 2.20, 95\% CI: 1.79-2.70 in women), delayed (OR: 2.04, 95\% CI: 1.62-2.57 in men; OR: 1.82, 95\% CI: $1.50-2.22$ in women), or genetic (OR: 1.70, 95\% CI: $1.32-2.20$ in men; OR: $1.51,95 \%$ CI: $1.21-1.89$ in women) effects were likely was positively associated with the worsened group (Table 2). In multivariate analysis, after controlling for individual and disaster-related factors, the significant associations for perceived radiation risk regarding immediate effects remained significant among women in the worsened group. On the other hand, having bad or very bad subjective health status, a history of psychiatric disease, a PCL-S score $\geq 44$, a history of cardiovascular disease among women, the experience of having heard the sound of the nuclear plant explosion among women, the experience of partial collapse or more severe damage to one's own home among women, and living in a dwelling other than one's own home were positively associated with the worsened group, whereas age $\geq 40$ years among men was negatively associated with the worsened group (Table 2).

\subsection{Predictors for the Recovered Group Compared with the Chronic Group}

Results from univariate and multivariate analyses of potential factors for the recovered group compared with the chronic group are shown in Table 3 (the results in total subjects are shown in Table S1). In univariate analysis, the perceived risk that immediate (OR: $0.75,95 \%$ CI: 0.57-0.97), delayed (OR: $0.72,95 \%$ CI: 0.53-0.98), or genetic (OR: 0.66, 95\% CI: 0.46-0.94) effects were very likely were negatively associated with the recovered group among men (Table 2).

Among women, the perceived risk that immediate effects were likely (OR: 0.76, 95\% CI: 0.61-0.94) or very likely (OR: $0.74,95 \%$ CI: 0.60-0.92) was negatively associated with the recovered group (Table 3 ). In multivariate analysis, after controlling for individual and disaster-related factors, the significant associations between perceived radiation risk and the recovered group became non-significant. On the other hand, having usual or better subjective health status, a PCL-S score $<44$, previous experience with tsunamis among women, living in Fukushima Prefecture among men, and living in one's own home among women were positively associated with the recovered group, whereas a history of psychiatric disease was negatively associated with the recovered group (Table 3). 
Table 2. Univariate and multivariate analysis by logistic regression comparing worsened with resistant group.

\begin{tabular}{|c|c|c|c|c|c|c|c|c|c|}
\hline & & \multicolumn{8}{|c|}{ Worsened $(K 6<13 \Rightarrow K 6 \geq 13)$ (Reference: Resistant; $K 6<13 \Rightarrow K 6<13$ ) } \\
\hline & & \multicolumn{4}{|c|}{ Univariate Analysis } & \multicolumn{4}{|c|}{ Multivariate Analysis } \\
\hline & & \multicolumn{2}{|c|}{ Men } & \multicolumn{2}{|c|}{ Women } & \multicolumn{2}{|c|}{ Men } & \multicolumn{2}{|c|}{ Women } \\
\hline & & OR $[95 \% \mathrm{CI}]$ & $p$ Value & OR $[95 \% \mathrm{CI}]$ & $p$ Value & OR $[95 \% \mathrm{CI}]$ & $p$ Value & OR $[95 \% \mathrm{CI}]$ & $p$ Value \\
\hline \multirow{3}{*}{ Age groups } & $15-39$ years & Reference & & Reference & & Reference & & Reference & \\
\hline & $40-64$ years & $0.80(0.65-0.97)$ & 0.008 & $1.09(0.92-1.28)$ & 0.122 & $0.70(0.56-0.89)$ & 0.003 & $0.84(0.69-1.02)$ & 0.072 \\
\hline & $\geq 65$ years & $0.96(0.78-1.17)$ & 0.365 & $1.44(1.22-1.70)$ & $<0.001$ & $0.75(0.59-0.97)$ & 0.025 & $0.82(0.67-1.02)$ & 0.068 \\
\hline \multirow{2}{*}{ Subjective health status } & Usual, good, or very good & Reference & & Reference & & Reference & & Reference & \\
\hline & Bad or very bad & $3.39(2.88-3.99)$ & $<0.001$ & $3.69(3.22-4.23)$ & $<0.001$ & $2.27(1.87-2.76)$ & $<0.001$ & $2.38(2.01-2.82)$ & $<0.001$ \\
\hline \multirow{2}{*}{ Past history } & $\begin{array}{l}\text { Cardiovascular disease (heart } \\
\text { disease and stroke) }\end{array}$ & $1.29(1.07-1.56)$ & 0.009 & $2.12(1.79-2.52)$ & $<0.001$ & $1.07(0.85-1.35)$ & 0.551 & $1.45(1.16-1.81)$ & 0.001 \\
\hline & Psychiatric disease & $3.19(2.40-4.23)$ & $<0.001$ & $3.73(3.02-4.61)$ & $<0.001$ & $1.92(1.38-2.67)$ & $<0.001$ & $2.58(2.02-3.30)$ & $<0.001$ \\
\hline \multirow{6}{*}{ Disaster experiences } & Tsunami & $1.27(1.08-1.49)$ & 0.004 & $1.41(1.21-1.64)$ & $<0.001$ & $1.00(0.82-1.22)$ & 1 & $0.95(0.78-1.15)$ & 0.576 \\
\hline & $\begin{array}{l}\text { heard the sound of nuclear } \\
\text { plant explosion }\end{array}$ & $1.59(1.36-1.85)$ & $<0.001$ & $1.79(1.57-2.03)$ & $<0.001$ & $1.13(0.94-1.36)$ & 0.194 & $1.26(1.08-1.46)$ & 0.004 \\
\hline & $\begin{array}{l}\text { bereavement } \\
\text { house damage }\end{array}$ & $1.37(1.15-1.63)$ & $<0.001$ & $1.40(1.21-1.62)$ & $<0.001$ & $1.05(0.85-1.28)$ & 0.662 & $1.01(0.85-1.20)$ & 0.936 \\
\hline & No damage & Reference & & Reference & & Reference & & Reference & \\
\hline & Partial collapse & $1.33(1.10-1.60)$ & 0.632 & $1.60(1.36-1.87)$ & 0.665 & $1.11(0.90-1.37)$ & 0.312 & $1.26(1.05-1.50)$ & 0.012 \\
\hline & Half collapse and worse & $1.62(1.21-2.17)$ & 0.01 & $2.40(1.91-3.01)$ & $<0.001$ & $0.97(0.69-1.37)$ & 0.86 & $1.50(1.14-1.97)$ & 0.004 \\
\hline \multirow{2}{*}{ Living place in 2011} & In Fukushima prefecture & Reference & & Reference & & Reference & & Reference & \\
\hline & Out of Fukushima prefecture & $1.46(1.22-1.74)$ & $<0.001$ & $1.13(0.980-1.31)$ & 0.092 & $1.22(0.99-1.50)$ & 0.064 & $0.96(0.80-1.14)$ & 0.616 \\
\hline \multirow{2}{*}{ Living arrangement in 2011} & Own home & Reference & & Reference & & Reference & & Reference & \\
\hline & Other than own home & $1.88(1.58-2.23)$ & $<0.001$ & $1.60(1.39-1.85)$ & $<0.001$ & $1.44(1.18-1.77)$ & $<0.001$ & $1.24(1.04-1.48)$ & 0.014 \\
\hline Posttraumatic stress symptoms & PCL-S score $\geq 44$ in 2011 & $5.84(4.97-6.86)$ & $<0.001$ & $5.26(4.61-6.02)$ & $<0.001$ & $4.04(3.32-4.90)$ & $<0.001$ & $3.35(2.85-3.95)$ & $<0.001$ \\
\hline \multirow{15}{*}{$\begin{array}{l}\text { Radiation Risk Perception in } \\
2011\end{array}$} & Immediate effect & & & & & & & & \\
\hline & Very unlikely & Reference & & Reference & & Reference & & Reference & \\
\hline & Unlikely & $1.46(1.21-1.76)$ & 0.013 & $1.58(1.36-1.84)$ & 0.042 & $0.98(0.78-1.22)$ & 0.839 & $1.32(1.10-1.58)$ & 0.003 \\
\hline & Likely & $2.33(1.82-2.99)$ & 0.003 & $2.20(1.79-2.70)$ & 0.01 & $1.14(0.84-1.55)$ & 0.41 & $1.34(1.03-1.74)$ & 0.028 \\
\hline & Very likely & $2.82(2.18-3.64)$ & $<0.001$ & $2.97(2.36-3.73)$ & $<0.001$ & $1.02(0.72-1.44)$ & 0.921 & $1.50(1.10-2.03)$ & 0.01 \\
\hline & Delayed effect & & & & & & & & \\
\hline & Very unlikely & Reference & & Reference & & Reference & & Reference & \\
\hline & Unlikely & $1.35(1.08-1.69)$ & $<0.001$ & $1.12(0.92-1.37)$ & $<0.001$ & $1.08(0.79-1.46)$ & 0.643 & $1.00(0.76-1.33)$ & 0.978 \\
\hline & Likely & $2.04(1.62-2.57)$ & 0.005 & $1.82(1.50-2.22)$ & $<0.001$ & $1.33(0.94-1.88)$ & 0.105 & $1.04(0.76-1.41)$ & 0.817 \\
\hline & Very likely & $2.96(2.38-3.67)$ & $<0.001$ & $2.31(1.90-2.80)$ & $<0.001$ & $1.32(0.90-1.92)$ & 0.155 & $0.95(0.68-1.32)$ & 0.755 \\
\hline & Genetic effect & & & & & & & & \\
\hline & Very unlikely & Reference & & Reference & & Reference & & Reference & \\
\hline & Unlikely & $1.23(0.95-1.61)$ & 0.001 & $0.88(0.69-1.12)$ & $<0.001$ & $1.11(0.78-1.56)$ & 0.568 & $0.73(0.53-1.01)$ & 0.055 \\
\hline & Likely & $1.70(1.32-2.20)$ & 0.18 & $1.51(1.21-1.89)$ & 0.019 & $1.08(0.75-1.57)$ & 0.681 & $1.04(0.75-1.44)$ & 0.828 \\
\hline & Very likely & $2.78(2.19-3.52)$ & $<0.001$ & $2.33(1.88-2.89)$ & $<0.001$ & $1.31(0.89-1.92)$ & 0.169 & $1.25(0.89-1.76)$ & 0.202 \\
\hline
\end{tabular}

OR, odds ratio. OR in multivariate analyses were adjusted for all other variables in table 
Table 3. Univariate and multivariate analysis by logistic regression comparing recovered with chronic group.

\begin{tabular}{|c|c|c|c|c|c|c|c|c|c|}
\hline & & \multicolumn{8}{|c|}{ Recovered (K6 $\geq 13 \Rightarrow K 6<13$ ) (Reference: Chronic; $K 6 \geq 13 \Rightarrow K 6 \geq 13$ ) } \\
\hline & & \multicolumn{4}{|c|}{ Univariate Analysis } & \multicolumn{4}{|c|}{ Multivariate Analysis } \\
\hline & & \multicolumn{2}{|c|}{ Men } & \multicolumn{2}{|c|}{ Women } & \multicolumn{2}{|c|}{ Men } & \multicolumn{2}{|c|}{ Women } \\
\hline & & OR $[95 \% \mathrm{CI}]$ & $p$ Value & OR $[95 \% \mathrm{CI}]$ & $p$ Value & OR $[95 \% \mathrm{CI}]$ & $p$ Value & OR $[95 \% \mathrm{CI}]$ & $p$ Value \\
\hline \multirow{3}{*}{ Age groups } & $15-39$ years & Reference & & Reference & & Reference & & Reference & \\
\hline & 40-64 years & $0.95(0.73-1.23)$ & 0.564 & $0.94(0.78-1.14)$ & 0.659 & $1.08(0.80-1.48)$ & 0.614 & $0.87(0.70-1.09)$ & 0.229 \\
\hline & $\geq 65$ years & $1.00(0.76-1.31)$ & 0.772 & $0.84(0.69-1.02)$ & 0.045 & $1.15(0.82-1.60)$ & 0.426 & $0.85(0.66-1.08)$ & 0.185 \\
\hline \multirow{2}{*}{ Subjective health status } & Bad or very bad & Reference & & Reference & & Reference & & Reference & \\
\hline & Usual, good, or very good & $1.90(1.57-2.30)$ & $<0.001$ & $2.11(1.83-2.43)$ & $<0.001$ & $1.70(1.36-2.13)$ & $<0.001$ & $1.61(1.36-1.91)$ & $<0.001$ \\
\hline \multirow{2}{*}{ Past history } & $\begin{array}{l}\text { Cardiovascular disease } \\
\text { (heart disease and stroke) }\end{array}$ & $0.97(0.77-1.21)$ & 0.754 & $0.73(0.61-0.89)$ & 0.001 & $1.08(0.82-1.41)$ & 0.59 & $0.91(0.72-1.16)$ & 0.454 \\
\hline & Psychiatric disease & $0.56(0.44-0.73)$ & $<0.001$ & $0.44(0.37-0.53)$ & $<0.001$ & $0.65(0.48-0.87)$ & 0.003 & $0.49(0.39-0.61)$ & $<0.001$ \\
\hline \multirow{6}{*}{ Disaster experiences } & Tsunami & $1.00(0.82-1.23)$ & 0.977 & $1.13(0.96-1.33)$ & 0.147 & $1.15(0.91-1.46)$ & 0.254 & $1.29(1.05-1.58)$ & 0.015 \\
\hline & $\begin{array}{l}\text { heard the sound of nuclear } \\
\text { plant explosion }\end{array}$ & $0.92(0.75-1.12)$ & 0.39 & $0.88(0.76-1.02)$ & 0.091 & $0.91(0.72-1.15)$ & 0.436 & $0.98(0.82-1.17)$ & 0.794 \\
\hline & $\begin{array}{l}\text { bereavement } \\
\text { house damage }\end{array}$ & $0.92(0.75-1.13)$ & 0.43 & $0.87(0.75-1.012)$ & 0.072 & $1.00(0.79-1.27)$ & 0.997 & $1.00(0.83-1.19)$ & 0.97 \\
\hline & No damage & Reference & & Reference & & Reference & & Reference & \\
\hline & Partial collapse & $0.96(0.74-1.25)$ & 0.17 & $1.01(0.84-1.22)$ & 0.142 & $1.03(0.77-1.38)$ & 0.825 & $1.11(0.90-1.36)$ & 0.342 \\
\hline & Half collapse and worse & $0.69(0.49-0.97)$ & 0.014 & $0.81(0.62-1.05)$ & 0.055 & $0.80(0.54-1.19)$ & 0.265 & $0.93(0.69-1.27)$ & 0.657 \\
\hline \multirow{2}{*}{ Living place in 2011} & Out of Fukushima prefecture & Reference & & Reference & & Reference & & Reference & \\
\hline & In Fukushima prefecture & $1.32(1.06-1.64)$ & 0.015 & $1.12(0.95-1.31)$ & 0.187 & $1.45(1.11-1.89)$ & 0.006 & $0.97(0.80-1.18)$ & 0.773 \\
\hline \multirow{2}{*}{ Living arrangement in 2011} & Other than own home & Reference & & Reference & & Reference & & Reference & \\
\hline & Own home & $1.21(0.96-1.52)$ & 0.099 & $1.40(1.18-1.66)$ & $<0.001$ & $1.03(0.78-1.35)$ & 0.849 & $1.29(1.05-1.59)$ & 0.016 \\
\hline Posttraumatic stress symptoms & PCL-S score < 44 in 2011 & $1.94(1.55-2.42)$ & $<0.001$ & $2.02(1.69-2.40)$ & $<0.001$ & $1.65(1.27-2.15)$ & $<0.001$ & $1.64(1.33-2.01)$ & $<0.001$ \\
\hline \multirow{15}{*}{$\begin{array}{l}\text { Radiation Risk Perception } \\
\text { in } 2011\end{array}$} & Immediate effect & & & & & & & & \\
\hline & Very unlikely & Reference & & Reference & & Reference & & Reference & \\
\hline & Unlikely & $0.86(0.68-1.09)$ & 0.883 & $0.98(0.82-1.17)$ & 0.05 & $0.99(0.75-1.31)$ & 0.93 & $1.03(0.84-1.27)$ & 0.777 \\
\hline & Likely & $0.89(0.67-1.19)$ & 0.789 & $0.76(0.61-0.94)$ & 0.104 & $1.12(0.79-1.60)$ & 0.529 & $0.79(0.61-1.03)$ & 0.085 \\
\hline & Very likely & $0.75(0.57-0.97)$ & 0.113 & $0.74(0.60-0.92)$ & 0.057 & $0.94(0.66-1.33)$ & 0.713 & $0.90(0.67-1.19)$ & 0.447 \\
\hline & Delayed effect & & & & & & & & \\
\hline & Very unlikely & Reference & & Reference & & Reference & & Reference & \\
\hline & Unlikely & $0.87(0.62-1.22)$ & 0.745 & $1.09(0.84-1.44)$ & 0.049 & $0.88(0.55-1.39)$ & 0.579 & $1.17(0.82-1.69)$ & 0.39 \\
\hline & Likely & $0.81(0.58-1.12)$ & 0.596 & $0.93(0.72-1.20)$ & 0.664 & $0.82(0.50-1.34)$ & 0.426 & $1.02(0.69-1.49)$ & 0.938 \\
\hline & Very likely & $0.72(0.53-0.98)$ & 0.034 & $0.82(0.64-1.04)$ & 0.005 & $0.93(0.55-1.56)$ & 0.781 & $1.06(0.71-1.57)$ & 0.784 \\
\hline & Genetic effect & & & & & & & & \\
\hline & Very unlikely & Reference & & Reference & & Reference & & Reference & \\
\hline & Unlikely & $0.86(0.57-1.31)$ & 0.761 & $1.11(0.78-1.59)$ & 0.272 & $0.90(0.53-1.54)$ & 0.697 & $0.93(0.59-1.48)$ & 0.765 \\
\hline & Likely & $0.85(0.58-1.25)$ & 0.834 & $1.13(0.81-1.58)$ & 0.109 & $1.09(0.63-1.87)$ & 0.768 & $1.10(0.69-1.76)$ & 0.686 \\
\hline & Very likely & $0.66(0.46-0.94)$ & 0.002 & $0.83(0.60-1.13)$ & $<0.001$ & $0.83(0.48-1.45)$ & 0.519 & $0.97(0.61-1.55)$ & 0.907 \\
\hline
\end{tabular}




\section{Discussion}

To the best of our knowledge, this is the first longitudinal study conducted to investigate the association between perceived radiation risk at baseline and mid-term mental health after a nuclear accident in a large sample. In this study, most (80.3\%) of the 36,056 evacuees were resistant to the disaster. This is consistent with a previous finding that few people develop chronic mental disorders after traumatic events [39]. Our results suggest that most sufferers are resistant to the disaster, even if disaster is complex, including an earthquake, tsunami, and nuclear accident. Notably, a greater proportion of the respondents were classified as being in the recovered group $(7.8 \%)$ compared with the worsened group (5.3\%). While the chronic stress resulting from the complex disaster worsened the mental health status of some of the participants, more showed resilience. Therefore, this study investigated predictive factors for both the recovered and the worsened groups.

We also found that the degree of perceived radiation risk predicted the degree of worsening mental health status after the disaster, although multivariate analysis revealed that the association between perceived radiation risk regarding immediate effects and the worsened group remained significant among only women after controlling for individual and disaster-related factors. This finding was consistent with that from a recent cross-sectional study demonstrating that perceived radiation risk was associated with psychological distress after the Fukushima disaster [18]. Previous studies investigating mental health after the Chernobyl nuclear accident found that poor mental health status could be explained by risk perception [20-22]; however, those studies were conducted at least 6.5 years after the nuclear accident. Interestingly, our results of the association between perceived radiation risk and the worsened group was observed among only women with regard to immediate effects after multivariate analysis. A recent meta-analysis demonstrated that being female was significantly associated with depression after natural disaster [11]. Furthermore, many studies suggested that women have greater tendency to express emotions such as fear after traumatic events $[40,41]$, and were less likely to use positive coping strategies [36]. Taken together, emotionality and coping strategies of women may account for our results of the association between perceived radiation risk regarding immediate effects and the worsened group among women. On the other hand, women might have more sensitive biological stress systems such as hypothalamic-pituitary-adrenal axis, which might have effects on the results. Boe et al. [5] investigated the association between pre-disaster vulnerability and increased risk of psychopathology, and found that a general neurotic personality predicted chronic psychopathology after a disaster. Although risk perception is influenced by disaster experiences [26], having a vulnerable personality may also affect the perception of radiation risk. Our results indicated that a perception of high radiation risk predicted worse psychological well-being after the disaster; this finding suggests that evacuees who believe that their health is highly affected by a nuclear accident are at a high risk of late-onset severe mental illness such as depression or anxiety disorder. Conversely, depressive symptoms among evacuees might influence their risk perception due to alternations in their cognitive processes, which is likely to be a vicious circle. Early detection and careful intervention are needed to prevent a deterioration of mental health among evacuees who believe that their health is highly affected by radiation. In particular, population scale intervention based on cognitive models of PTSD [19] may be helpful to reduce risk perception among vulnerable groups [42]. Although risk communication is challenging, it is necessary in order to help evacuees make well-informed decisions about life changes [43].

Having PTSD symptoms related to the nuclear disaster at baseline was significantly associated with mental health status during the 2-year period of this study; this finding was consistent with those from previous study [9] reporting that the presence of PTSD symptoms at baseline predicted poor mid-term mental health. Moreover, it is well known that depression often coexists with PTSD [1]. Similarly, a history of psychiatric disease was strong predictor for psychological distress. Although we did not assess the diagnosis of the psychiatric disease, it is estimated that patients with neurotic disorder might have fear and anxiety concerning disasters, which leads to persistent anticipatory anxiety [44]. Those who have a psychiatric disease have more vulnerability to distress [4], and were 
more strongly affected by forced evacuations and other similar experiences after the Fukushima disaster, which was a complex disaster involving a nuclear accident. Taken together, the coexistence of PTSD or past psychiatric disease were strong predictors of poor mid-term mental health. Since complex disasters have various effects on the lives of evacuees, including secondary effects such as new living arrangements or changes in job status, the presence of trauma and vulnerability to distress should be assessed from the early phase after a disaster.

Our results regarding the positive association between the worsened group and not living in one's own home are consistent with those from a previous systematic review [45] reporting that relocated individuals were more likely to experience psychological distress or depression after a disaster. Interestingly, living in Fukushima Prefecture in 2011 was a significant predictor among men in the recovered group, whereas no such association was observed among women. Although no association was found between living outside of Fukushima Prefecture and psychological distress at baseline [18], living outside of Fukushima Prefecture may cause various changes in daily life, such as in job or financial status, especially among men. Our results suggest that place of residence may affect mid-term psychological well-being, and thus outreach services are needed to provide careful intervention not only inside, but also outside the prefecture.

This study did have several limitations. First, a relatively low proportion of the target population was included in the analysis, which might have affected the interpretation of the results. Previous studies $[46,47]$ showed that the mental health status might have effects on response rate to survey, suggesting that non-response was associated with bad mental health status. There might be many residents who were in a bad condition and could not answer the survey. This is important limitation of this study. Second, insufficient information was available regarding pre-disaster factors such as personality traits, social adaptation traumatic experiences, or other mental health problems including addictive behavior. Third, the several risk factors in this study are general risk factors, and may be related to general mental health rather than disaster-specific problems. Finally, the baseline survey was conducted 10 months after the disaster, which is a relatively long time because the baseline might include subsequent changes that do not reflect the status of the evacuees just after the disaster. Additionally, we did not assess whether the subjects received psychological interventions. Nevertheless, to our knowledge, this was the first longitudinal study on the association between perceived radiation risk and mid-term mental health after a nuclear accident. Our finding that a perception of high radiation risk is a predictor for worse mid-term mental health in women is expected to contribute to the assessment of and interventions for evacuees after nuclear accidents.

\section{Conclusions}

In summary, we found a significant association between perceived radiation risk regarding immediate effects and mid-term mental health after a nuclear accident in women, indicating that female evacuees who believe that their health is highly affected by a nuclear accident are at an increased risk of poor mid-term mental health. Although it is difficult to generalize these findings to other disasters, the assessment of perceived risk and other predictors such as PTSD symptoms or a history of psychiatric disease is needed in order to promote careful intervention for affected populations.

Supplementary Materials: The following are available online at www.mdpi.com/1660-4601/14/9/1067/s1, Figure S1: Flow chart of the study selection, Table S1: Univariate and multivariate analysis by logistic regression in total subjects.

Author Contributions: Itaru Miura, Masato Nagai, Masaharu Maeda, Misari Oe, Yuriko Suzuki, Hideto Takahashi, Tetsuya Ohira, Seiji Yasumura conceived and designed the study. Masato Nagai, Mayumi Harigane, Hideto Takahashi, Tetsuya Ohira obtained and analyzed the data. Itaru Miura, Masato Nagai, Masaharu Maeda, Misari Oe, Hideto Takahashi, Tetsuya Ohira, Seiji Yasumura wrote the first draft of the paper. Itaru Miura, Masato Nagai, Masaharu Maeda, Mayumi Harigane, Senta Fujii, Misari Oe, Hirooki Yabe, Yuriko Suzuki, Hideto Takahashi, Tetsuya Ohira, Seiji Yasumura, Masafumi Abe edited and revised the paper.

Conflicts of Interest: The authors declare no conflict of interest. 


\section{References}

1. Foa, E.B.; Stein, D.J.; McFarlane, A.C. Symptomatology and psychopathology of mental health problems after disaster. J. Clin. Psychiatry 2006, 67 (Suppl. 2), 15-25. [PubMed]

2. McFarlane, A.C. The longitudinal course of posttraumatic morbidity. The range of outcomes and their predictors. J. Nerv. Ment. Dis. 1988, 176, 30-39. [CrossRef] [PubMed]

3. Carr, V.J.; Lewin, T.J.; Webster, R.A.; Kenardy, J.A.; Hazell, P.L.; Carter, G.L. Psychosocial sequelae of the 1989 Newcastle earthquake: II. Exposure and morbidity profiles during the first 2 years post-disaster. Psychol. Med. 1997, 27, 167-178. [CrossRef] [PubMed]

4. Norris, F.H.; Perilla, J.L.; Riad, J.K.; Kaniasty, K.; Lavizzo, E.A. Stability and change in stress, resources, and psychological distress following natural disaster: Findings from hurricane Andrew. Anxiety Stress Coping 1999, 12, 363-396. [CrossRef] [PubMed]

5. Boe, H.J.; Holgersen, K.H.; Holen, A. Mental health outcomes and predictors of chronic disorders after the North Sea oil rig disaster: 27-year longitudinal follow-up study. J. Nerv. Ment. Dis. 2011, 199, 49-54. [CrossRef] [PubMed]

6. Van der Velden, P.G.; Wong, A.; Boshuizen, H.C.; Grievink, L. Persistent mental health disturbances during the 10 years after a disaster: Four-wave longitudinal comparative study. Psychiatry Clin. Neurosci. 2013, 67, 110-118. [CrossRef] [PubMed]

7. Fergusson, D.M.; Horwood, L.J.; Boden, J.M.; Mulder, R.T. Impact of a major disaster on the mental health of a well-studied cohort. JAMA Psychiat. 2014, 71, 1025-1031. [CrossRef] [PubMed]

8. Chen, Y.L.; Hsu, W.Y.; Lai, C.S.; Tang, T.C.; Wang, P.W.; Yeh, Y.C.; Huang, M.F. Yen, C.F.; Chen, C.S. One-year follow up of PTSD and depression in elderly aboriginal people in Taiwan after Typhoon Morakot. Psychiatry Clin. Neurosci. 2013, 69, 12-21. [CrossRef] [PubMed]

9. Neria, Y.; Olfson, M.; Gameroff, M.J.; DiGrande, L.; Wickramaratne, P.; Gross, R.; Pilowsky, D.J.; Neugebaur, R.; Manetti-Cusa, J.; Lewis-Fernandez, R.; et al. Long-term course of probable PTSD after the 9/11 attacks: A study in urban primary care. J. Trauma. Stress 2010, 23, 474-482. [CrossRef] [PubMed]

10. Fernandez, A.; Black, J.; Jones, M.; Wilson, L.; Salvador-Carulla, L.; Astell-Burt, T.; Black, D. Flooding and mental health: A systematic mapping review. PLoS ONE 2015, 10, e0119929.

11. Tang, B.; Liu, X.; Liu, Y.; Xue, C.; Zhang, L. A meta-analysis of risk factors for depression in adults and children after natural disasters. BMC Public Health 2014, 14, 623. [CrossRef] [PubMed]

12. Ginzburg, H.M. The psychological consequences of the Chernobyl accident e findings from the International Atomic Energy Agency study. Public Health Rep. 1993, 108, 184-192. [PubMed]

13. Viinamäki, H.; Kumpusalo, E.; Myllykangas, M.; Salomaa, S.; Kumpusalo, L.; Kolmakov, S.; Ilchenko, I.; Zhukowsky, G.; Nissinen, A. The Chernobyl accident and mental wellbeing e a population study. Acta Psychiatr. Scand. 1995, 91, 396-401. [CrossRef] [PubMed]

14. Havenaar, J.M.; Rumyantzeva, G.M.; van den Brink, W.; Poelijoe, N.W.; van den Bout, J.; van Engeland, H.; Koeter, M.W. Longterm mental health effects of the Chernobyl disaster: An epidemiologic survey in two former Soviet regions. Am. J. Psychiatry 1997, 154, 1605-1607. [CrossRef] [PubMed]

15. Foster, R.P.; Goldstein, M.F. Chernobyl disaster sequelae in recent immigrants to the United States from the former Soviet Union (FSU). J. Immigr. Minor. Health 2007, 9, 115-124. [CrossRef] [PubMed]

16. Adams, R.E.; Bromet, E.J.; Panina, N.; Golovakha, E. Stress and wellbeing in mothers of young children 11 years after the Chornobyl nuclear power plant accident. Psychol. Med. 2002, 32, 143-156. [CrossRef] [PubMed]

17. Bromet, E.J.; Gluzman, S.; Schwartz, J.E.; Goldgaber, D. Somatic symptoms in women 11 years after the Chornobyl accident. Environ. Health Perspect. 2002, 110 (Suppl. 4), 625-629. [CrossRef] [PubMed]

18. Suzuki, Y.; Yabe, H.; Yasumura, S.; Ohira, T.; Niwa, S.; Ohtsuru, A.; Mashiko, H.; Maeda, M.; Abe, M.; Mental Health Group of the Fukushima Health Management Survey. Psychological distress and the perception of radiation risks: The Fukushima health management survey. Bull. World Health Organ. 2015, 93, 598-605. [CrossRef] [PubMed]

19. Ehlers, A.; Clark, D.M. A cognitive model of posttraumatic stress disorder. Behav. Res. Therapy 2000, 38, 319-345. [CrossRef]

20. Havenaar, J.M.; de Wilde, E.J.; van den Bout, J.; Drottz-Sjöberg, B.M.; van den Brink, W. Perception of risk and subjective health among victims of the Chernobyl disaster. Soc. Sci. Med. 2003, 56, 569-572. [CrossRef] 
21. Adams, R.E.; Guey, L.T.; Gluzman, S.F.; Bromet, E.J. Psychological well-being and risk perceptions of mothers in Kyiv, Ukraine, 19 years after the Chornobyl disaster. Int. J. Soc. Psychiatry 2011, 57, 637-645. [PubMed]

22. Bromet, E.J. Emotional consequences of nuclear power plant disasters. Health Phys. 2014, 106, $206-210$. [CrossRef] [PubMed]

23. Mills, M.A.; Edmondson, D.; Park, C.L. Trauma and stress response among Hurricane Katrina evacuees. Am. J. Public Health 2007, 97 (Suppl. 1), S116-S123. [CrossRef] [PubMed]

24. Cukor, J.; Wyka, K.; Jayasinghe, N.; Weathers, F.; Giosan, C.; Leck, P.; Roberts, J.; Spielman, L.; Crane, M.; Difede, J.; et al. Prevalence and predictors of posttraumatic stress symptoms in utility workers deployed to the World Trade Center following the attacks of 11 September 2001. Depress. Anxiety 2011, 28, 210-217. [PubMed]

25. Kim, Y.; Tsutsumi, A.; Izutsu, T.; Kawamura, N.; Miyazaki, T.; Kikkawa, T. Persistent distress after psychological exposure to the Nagasaki atomic bomb explosion. Br. J. Psychiatry 2011, 199, 411-416. [CrossRef] [PubMed]

26. Ho, M.C.; Shaw, D.; Lin, S.; Chiu, Y.C. How do disaster characteristics influence risk perception? Risk Anal. 2008, 28, 635-643. [PubMed]

27. Yasumura, S.; Hosoya, M.; Yamashita, S.; Kamiya, K.; Abe, M.; Akashi, M.; Kodama, K.; Ozasa, K.; Fukushima Health Management Survey Group. Study protocol for the Fukushima health management survey. J. Epidemiol. 2012, 22, 375-383. [CrossRef] [PubMed]

28. Yabe, H.; Suzuki, Y.; Mashiko, H.; Nakayama, Y.; Hisata, M.; Niwa, S.; Yasumura, S.; Yamashita, S.; Kamiya, K.; Abe, M.; et al. Psychological distress after the great East Japan earthquake and Fukushima Daiichi nuclear power plant accident: Results of a mental health and lifestyle survey through the Fukushima health management survey in FY2011 and FY2012. Fukushima J. Med. Sci. 2014, 60, 57-67. [CrossRef] [PubMed]

29. Kessler, R.C.; Barker, P.R.; Colpe, L.J.; Epstein, J.F.; Gfroerer, J.C.; Hiripi, E.; Howes, M.J.; Normand, S.L.; Manderscheid, R.W.; Walters, E.E.; et al. Screening for serious mental illness in the general population. Arch. Gen. Psychiatry 2003, 60, 184-189. [PubMed]

30. Sakurai, K.; Nishi, A.; Kondo, K.; Yanagida, K.; Kawakami, N. Screening performance of K6/K10 and other screening instruments for mood and anxiety disorders in Japan. Psychiatry Clin. Neurosci. 2011, 65, 434-441. [PubMed]

31. Kessler, R.C.; Galea, S.; Gruber, M.J.; Sampson, N.A.; Ursano, R.J.; Wessely, S. Trends in mental illness and suicidality after Hurricane Katrina. Mol. Psychiatry 2008, 13, 374-384. [PubMed]

32. Blanchard, E.B.; Jones-Alexander, J.; Buckley, T.C.; Forneris, C.A. Psychometric properties of the PTSD Checklist (PCL). Behav. Res. Ther. 1996, 34, 669-673. [PubMed]

33. Iwasa, H.; Suzuki, Y.; Shiga, T.; Maeda, M.; Yabe, S.; Yasumura, S.; Mental Health Group of the Fukushima health management survey. Psychometric evaluation of the Japanese version of the posttraumatic stress disorder checklist in community dwellers following the Fukushima Daiichi Nuclear Power Plant incident: The Fukushima Health Management Survey. SAGE Open 2016, 6, 1-11.

34. Lindell, M.K.; Barnes, V.E. Protective response to technological emergency: Risk perception and behavioral intention. Nucl. Saf. 1986, 27, 457-467.

35. Council for International Organizations of Medical Sciences. International guidelines for ethical review of epidemiological studies. Law Med. Health Care 1991, 19, 247-258.

36. Dai, W.; Chen, L.; Lai, Z.; Li, Y.; Wang, J.; Liu, A. The incidence of post-traumatic stress disorder among survivors after earthquakes: A systematic review and meta-analysis. BMC Psychiatry 2016, 16, 188.

37. Tolin, D.F.; Foa, E.B. Sex differences in trauma and posttraumatic stress disorder: A quantitative review of 25 years of research. Psychol. Bull. 2006, 132, 959-992. [PubMed]

38. Ministry of Health, Labour and Welfare, Japan. Comprehensive Survey of Living Conditions, 2010. Available online: http://www.mhlw.go.jp/toukei/saikin/hw/k-tyosa/k-tyosa10/ (accessed on 29 March 2016). (In Japanese)

39. Friedman, M.J.; Ritchie, E.C.; Watson, P.J. Overview, In Interventions Following Mass Violence and Disasters: Strategies for Mental Health Practice; Ritchie, E.C., Watson, P.J., Friedman, M.J., Eds.; The Guilford Press: New York, NY, USA, 2006; pp. 3-15.

40. Anderson, K.M.; Manuel, G. Gender differences in reported stress response to the Loma Prieta earthquake. Sex Roles 1994, 30, 725-733. 
41. Lilly, M.M.; Pole, N.; Best, S.R.; Metzler, T.; Marmar, C.R. Gender and PTSD: What can we learn from female police officers? J. Anxiety Disord. 2009, 23, 767-774. [PubMed]

42. Ehlers, A.; Clark, D.M.; Hackmann, A.; McManus, F.; Fennell, M. Cognitive therapy for post-traumatic stress disorder: development and evaluation. Behav. Res. Therapy 2005, 43, 413-431.

43. Ohtsuru, A.; Tanigawa, K.; Kumagai, A.; Niwa, O.; Takamura, N.; Midorikawa, S.; Nollet, K.; Yamashita, S.; Ohto, H.; Chhem, R.K.; et al. Nuclear disasters and health: Lessons learned, challenges, and proposals. Lancet 2015, 386, 489-497. [CrossRef]

44. Inoue, K.; Suda, S.; Shioda, K.; Kobayashi, T.; Kishi, K.; Kato, S. Differences in vulnerability to traumatic stress among patients with psychiatric disorders: One-year follow-up study after the Great East Japan Earthquake. Psychiatry Clin. Neurosci. 2015, 69, 587-595. [CrossRef] [PubMed]

45. Uscher-Pines, L. Health effects of relocation following disaster: A systematic review of the literature. Disasters 2009, 33, 1-22. [CrossRef] [PubMed]

46. Kaerlev, L.; Kolstad, H.A.; Hansen, A.M.; Thomsen, J.F.; Kærgaard, A.; Rugulies, R.; Mikkelsen, S.; Andersen, J.H.; Mors, O.; Grynderup, M.B.; et al. Are risk estimates biased in follow-up studies of psychosocial factors with low base-line participation? BMC Public Health 2011, 11, 539. [CrossRef] [PubMed]

47. Vercambre, M.N.; Gilbert, F. Respondents in an epidemiologic survey had fewer psychotropic prescriptions than nonrespondents: An insight into health-related selection bias using routine health insurance data. J. Clin. Epidemiol. 2012, 65, 1181-1189. [CrossRef] [PubMed]

(C) 2017 by the authors. Licensee MDPI, Basel, Switzerland. This article is an open access article distributed under the terms and conditions of the Creative Commons Attribution (CC BY) license (http://creativecommons.org/licenses/by/4.0/). 